\title{
Predictive Performance of a Vancomycin Population Pharmacokinetic Model in Neonates
}

\author{
Chris Stockmann (D) Adam L. Hersh · Jessica K. Roberts • \\ Jiraganya Bhongsatiern · Ernest K. Korgenski • Michael G. Spigarelli • \\ Catherine M. T. Sherwin · Adam Frymoyer
}

To view enhanced content go to www.infectiousdiseases-open.com

Received: March 31, 2015 / Published online: May 22, 2015

(C) The Author(s) 2015. This article is published with open access at Springerlink.com

\begin{abstract}
Introduction: The pharmacokinetics of vancomycin are highly variable among neonates, which makes dosing challenging in this population. However, adequate drug exposure is critical, especially when treating methicillin-resistant Staphylococcus aureus

Electronic supplementary material The online version of this article (doi:10.1007/s40121-015-0067-9) contains supplementary material, which is available to authorized users.
\end{abstract}

C. Stockmann · J. K. Roberts · M. G. Spigarelli

C. M. T. Sherwin

Division of Clinical Pharmacology, Department of Pediatrics, University of Utah School of Medicine, Salt Lake City, UT, USA

C. Stockmann $(\varangle) \cdot$ A. L. Hersh

Division of Pediatric Infectious Diseases,

Department of Pediatrics, University of Utah School

of Medicine, Salt Lake City, UT, USA

e-mail: Chris.Stockmann@hsc.utah.edu

J. Bhongsatiern

James L. Winkle College of Pharmacy, University of Cincinnati, Cincinnati, OH, USA

E. K. Korgenski

Pediatric Clinical Program, Intermountain

Healthcare, Salt Lake City, UT, USA

A. Frymoyer

Department of Pediatrics, Stanford University,

Palo Alto, CA, USA
(MRSA) infections. Utilization of population pharmacokinetic models and Bayesian methods offers the potential for developing individualized therapeutic approaches. To meet this need, a neonatal vancomycin population pharmacokinetic model was recently published. The current study sought to externally evaluate the predictive performance and generalizability of this model. Methods: A retrospective chart review of neonates who received vancomycin and had $\geq 1$ peak and $\geq 1$ trough concentrations at five Intermountain Healthcare neonatal intensive care units from 2006 to 2013 was performed and served as the external validation cohort. The published population pharmacokinetic model was implemented in NONMEM 7.2 with the structural and variance parameter values set equal to the estimates reported previously. The model was then used to predict the first peak and trough concentration for each neonate in the validation cohort and the model prediction error and absolute prediction error were calculated. Normalized prediction distribution errors (NPDE) were also evaluated.

Results: A total of 243 neonates were studied with a median postmenstrual age of 33 (range: 
23-54) weeks and a median weight of 1.6 (range: $0.4-6.8) \mathrm{kg}$. The model predicted the observed vancomycin concentrations with reasonable precision. For all vancomycin concentrations, the median prediction error was -0.8 (95\% CI: -1.4 to -0.4$) \mathrm{mg} / \mathrm{L}$ and the median absolute prediction error was 3.0 (95\% CI: 2.7-3.5) $\mathrm{mg} / \mathrm{L}$. No trends in NPDE across weight, postmenstrual age, serum creatinine, or time after dose were observed.

Conclusion: An evaluation of a recently published neonatal vancomycin population pharmacokinetic model in a large external dataset supported the predictive performance and generalizability of the model. This model may be useful in evaluating neonatal vancomycin dosing regimens and estimating the extent of drug exposure.

Keywords: Infectious diseases; MRSA; Neonates; Pharmacokinetics; Staphylococcus aureus; Vancomycin

\section{INTRODUCTION}

Optimizing vancomycin dosing to rapidly achieve adequate drug exposure is imperative in treating neonatal sepsis, particularly when treating invasive methicillin-resistant Staphylococcus aureus (MRSA) infections [1]. However, this has been challenging in neonates as the pharmacokinetics of vancomycin are highly variable among neonates due to developmental and pathophysiological changes [2, 3]. Recent studies have shown that standard neonatal vancomycin dosing strategies, such as those outlined in NeoFax ${ }^{\circledR}$ (Truven Health Analytics), do not reliably achieve trough concentrations $>10 \mathrm{mg} / \mathrm{L}[4,5]$. In addition, the ratio of the $24-\mathrm{h}$ area under the concentration-time curve $\left(\mathrm{AUC}_{24}\right)$ to the minimum inhibitory concentration (MIC)the best predictor of successful outcomes when treating invasive MRSA infections-is not routinely utilized to assess the appropriateness of vancomycin dosing in neonates, presumably due to practical limitations associated with calculating the $\mathrm{AUC}_{24}$.

Innovative vancomycin dosing strategies are therefore needed in neonates that: (1) incorporate known patient-specific determinants of vancomycin pharmacokinetics such as size, maturation, and renal function in the dose selection, and (2) allow for assessment of $\mathrm{AUC}_{24}$ based on the dosing history and vancomycin concentration(s) measured as part of routine therapeutic drug monitoring $[3,6,7]$. To develop such an individualized therapeutic approach in neonates, utilization of population pharmacokinetic models and Bayesian methods will be essential [8-11]. We recently developed a neonatal vancomycin population pharmacokinetic model that capitalized on patient data readily available in the electronic medical record: weight (an indicator of size), postmenstrual age (an indicator of maturation), and serum creatinine (an indicator of renal function) [7]. The model has the potential to improve our ability to define vancomycin dosing regimens that reliably achieve recommended exposure targets; however, it is critical to first evaluate whether this model and its findings are generalizable to neonates outside of the original population used to develop the model. The objective of the current study was to conduct an external evaluation of this published pharmacokinetic model and to enhance our understanding of the relationship between vancomycin trough concentration and $\mathrm{AUC}_{24}$ in neonates. 


\section{METHODS}

\section{Validation Cohort}

Approval to conduct this study was granted by the University of Utah and Primary Children's Hospital (PCH) Institutional Review Boards. $\mathrm{PCH}$ is a freestanding children's hospital with a level IV neonatal intensive care unit that is staffed by University of Utah neonatologists. $\mathrm{PCH}$ is owned and operated by Intermountain Healthcare, which is a large, not-for-profit, vertically integrated healthcare delivery system that serves Utah, Idaho, Wyoming, Nevada, and Montana. In addition to $\mathrm{PCH}$, four other level II-III neonatal intensive care units operated by Intermountain Healthcare were included in this study.

A retrospective chart review was conducted for all neonates who had vancomycin therapeutic drug monitoring performed from 2006 to 2013 at five Intermountain Healthcare neonatal intensive care units. Neonates were included if they were $<54$ weeks postmenstrual age and had $\geq 2$ doses of vancomycin, $\geq 1$ peak concentration, $\geq 1$ trough concentration, and $\geq 1$ serum creatinine level. Vancomycin concentrations were quantified using a particle-enhanced turbidimetric inhibition immunoassay on an Abbott Architect System platform (Abbott Laboratories, Abbott Park, IL, USA). Vancomycin concentrations were defined based on their temporal relationship to dosing records. Trough concentrations were defined as concentrations obtained within $3 \mathrm{~h}$ of the next vancomycin dose and peak concentrations were defined as concentrations obtained within $3 \mathrm{~h}$ of the preceding dose. Serum creatinine levels collected within $\pm 48 \mathrm{~h}$ of vancomycin dosing and concentration records were carried forward and backward and were used in the analyses. To account for the known difference in measured serum creatinine concentrations between the Jaffe method (used in the original model derivation cohort) and the enzymatic method (used in the current external validation cohort), a previously described linear conversion factor was applied to all of the enzymatic serum creatinine concentrations included in this external validation (enzymatic concentration $=1.050 \times$ Jaffe method concentration -0.122 ) [12]. Exclusion criteria included a diagnosis of congenital kidney disease, major congenital heart disease (other than ventricular septal defect, atrial septal defect, or patent ductus arteriosus), or extracorporeal membrane oxygenation during the vancomycin course.

\section{Model Evaluation}

The published neonatal vancomycin population pharmacokinetic model was implemented in the non-linear mixed effects modeling software NONMEM 7.2 (ICON Development Solutions, Ellicott City, MD, USA) as previously described [7]. Briefly, a one compartment model with first-order elimination was used to describe vancomycin pharmacokinetics. Clearance (CL) was predicted by weight (an indicator of size), postmenstrual age (PMA; an indicator of maturation) and serum creatinine $(\mathrm{Cr}$; an indicator of renal function) according to the following equation:

$$
\begin{aligned}
\mathrm{CL}(\mathrm{L} / \mathrm{h})= & 0.345 \times\left(\frac{\text { Weight }}{2.9 \mathrm{~kg}}\right)^{0.75} \\
& \times \frac{1}{1+\left(\frac{\mathrm{PMA}_{\text {weeks }}}{34.8}\right)^{-4.53}} \times\left(\frac{1}{\mathrm{Cr}_{\mathrm{mg} / \mathrm{dL}}}\right)^{0.267}
\end{aligned}
$$

Volume of distribution (V) was predicted by weight: 


$$
\mathrm{V}(\mathrm{L})=1.75 \times\left(\frac{\text { Weight }}{2.9 \mathrm{~kg}}\right)
$$

After accounting for known predictors, the remaining variation between neonates was described by an exponential error model for both CL (\% coefficient of variation [\% CV] $21.6 \%$ ) and V (\% CV 10.9\%). Residual variability (a measure of the difference between the model predicted concentration for a neonate and the observed concentration in that neonate) was captured using a combined proportional (\% CV 20.5\%) and additive error model (standard deviation $[\mathrm{SD}] \pm 1.3 \mathrm{mg} / \mathrm{L}$ ).

For each neonate in the external validation cohort, vancomycin concentrations were then predicted using the parameters of the population pharmacokinetic model and simulating the actual dosing regimen given to the neonate (using the NONMEM MAXEVAL $=0$ POSTHOC command). Only concentrations at times for which a neonate had therapeutic drug monitoring performed were simulated. Model predicted vancomycin concentrations (PRED from the NONMEM output) were then compared with the corresponding observed vancomycin concentrations. As described by Sheiner and Beal [13], the bias and precision of the model were assessed by calculating the median prediction error and median absolute prediction error for the first trough and peak concentration according to the following formulas:

Prediction error (bias): $\left(\frac{\text { Conc }_{\text {pred }}-\text { Conc }_{\mathrm{obs}}}{\text { Conc }_{\mathrm{obs}}}\right)$,

Absolute prediction error (precision):

$$
\left(\frac{\mid \text { Conc }_{\text {pred }}-\text { Conc }_{\text {obs }} \mid}{\text { Conc }_{\text {obs }}}\right) \text {, }
$$

where Conc $_{\text {pred }}$ refers to the model predicted vancomycin concentration and Conc $_{\mathrm{obs}}$ refers to the observed vancomycin concentration. Model predicted vancomycin concentrations calculated using each patient's individual Bayesian estimate of CL and V (i.e., the IPRED from the NONMEM output, which incorporates the patient's drug concentrations in addition to the fixed covariate effects in the model predictions) were also evaluated using the same approach.

The predictive performance of the model was further evaluated using simulation-based diagnostic methods. Normalized prediction distribution errors (NPDE) were calculated by simulating 1,000 data sets and comparing the predicted concentrations to the observed concentrations using the NPDE command in NONMEM $[14,15]$. The NPDE should follow a normal distribution with a theoretical mean of 0 and variance equal to 1 [14].

\section{Trough Concentration and $\mathrm{AUC}_{24}$ Relationship}

Following model evaluation, the relationship between trough concentration and $\mathrm{AUC}_{24}$ was examined. Bayesian estimates of $\mathrm{CL}$ for each neonate from the population pharmacokinetic model were used to calculate $\mathrm{AUC}_{24}$ at the time that vancomycin trough concentrations were collected [8]. $\mathrm{AUC}_{24}$ was calculated as the daily dose $\div$ CL. For a given trough concentration, the proportion of neonates with that trough concentration who achieved an $\mathrm{AUC}_{24} \geq 400$ was calculated. An $\mathrm{AUC}_{24} \geq 400 \mathrm{mg} \times \mathrm{hr} / \mathrm{L}$ would predict an $\mathrm{AUC}_{24} / \mathrm{MIC} \geq 400$ for an $\mathrm{MIC}$ of $\leq 1 \mathrm{mg} / \mathrm{L}$. $\mathrm{AUC}_{24}$ calculations, descriptive statistics, and graphical analyses were 
performed in $\mathrm{R}$ 3.1.1 ( $\mathrm{R}$ Foundation for Statistical Computing, Vienna, Austria).

\section{RESULTS}

\section{External Validation Cohort}

Overall, 243 neonates had vancomycin dose and concentration data available and served as the external validation cohort (see Fig. S1 in the electronic supplementary material [ESM]). The median dose was 15.5 (interquartile range [IQR]: $13.9-19.3) \mathrm{mg} / \mathrm{kg}$ and the median dosing interval was 11.5 (IQR: 8.0-12.5) h. Demographic and clinical characteristics of the neonates in the external validation cohort are shown in Table 1. For comparison, demographic and clinical characteristics of the neonates in the cohort used to develop the original published pharmacokinetic model are also shown. Overall, the validation cohort was of lower weight and age and had higher serum creatinine concentrations.

In the external validation cohort, a total of 734 vancomycin concentrations were available for analysis. Each neonate contributed a mean of $3.0( \pm 1.8)$ vancomycin concentrations. The time of vancomycin concentration collection relative to the previous dose is shown in Table 2. All neonates had at least one concentration measured within three hours of the end of the vancomycin infusion. No concentrations were below the lower limit of quantitation.

\section{Model Evaluation}

The vancomycin pharmacokinetic model adequately described the observed vancomycin concentrations in the external cohort of

Table 1 Demographic and clinical characteristics of neonates who received vancomycin and had therapeutic drug monitoring performed

\begin{tabular}{|c|c|c|c|c|}
\hline \multirow[t]{2}{*}{ Characteristic } & \multicolumn{2}{|c|}{ Model development cohort $(n=249)^{a}$} & \multicolumn{2}{|c|}{ External validation cohort $(n=243)^{b}$} \\
\hline & Median/No. & Range & Median/No. & Range \\
\hline Female, $n(\%)$ & $121(49 \%)$ & - & $103(42 \%)$ & - \\
\hline Gestational age, weeks & 34 & $23-42$ & 30 & $22-41$ \\
\hline Birth weight, kg & 2.0 & $0.4-4.4$ & 1.3 & $0.5-5.1$ \\
\hline Weight, kg & 2.9 & $0.5-6.3$ & 1.6 & $0.4-6.8$ \\
\hline Postnatal age, days & 19 & $0-173$ & 12 & $0-196$ \\
\hline Postmenstrual age, weeks & 39 & $24-53$ & 33 & $23-54$ \\
\hline APGAR at $5 \mathrm{~min}$ & 8 & $1-10$ & 8 & $1-10$ \\
\hline Serum creatinine, $\mathrm{mg} / \mathrm{dL}^{\mathrm{d}}$ & 0.4 & $0.1-2.7$ & 0.6 & $0.3-1.5$ \\
\hline
\end{tabular}

APGAR Newborn scale based on Appearance, Pulse, Grimace, Activity, and Respiration

a Patient characteristics of the 249 neonates used to develop the neonatal vancomycin population pharmacokinetic model described by Frymoyer et al. [7]

b Patient characteristics of the 243 neonates used in the current external validation

c The serum creatinine concentration in the model derivation cohort was measured using the Jaffe method. The serum creatinine concentration in the external validation cohort was measured using the enzymatic method and was converted to a Jaffe-standardized equivalent using a linear equation described by Srivastava et al. [12]. Converted values are presented in the table above 
neonates (Fig. 1a). Model predicted vancomycin concentrations (PRED) were slightly lower than the observed concentrations (median prediction error $-0.8 \quad[95 \%$ CI: -1.4 to $-0.4] \mathrm{mg} / \mathrm{L})$. The precision of the model was

Table 2 Timing of 734 neonatal vancomycin concentrations relative to the end of the most recent $1-\mathrm{h}$ infusion

\begin{tabular}{lc}
\hline $\begin{array}{l}\text { Time since the end of the most recent } \\
\text { infusion (h) }\end{array}$ & $\boldsymbol{N}$ (\%) \\
\hline $0-1$ & $122(17 \%)$ \\
$1-2$ & $192(26 \%)$ \\
$2-4$ & $27(4 \%)$ \\
$4-6$ & $63(9 \%)$ \\
$6-8$ & $66(9 \%)$ \\
$8-12$ & $152(21 \%)$ \\
$12-24$ & $107(15 \%)$ \\
$>24$ & $5(1 \%)$ \\
\hline
\end{tabular}

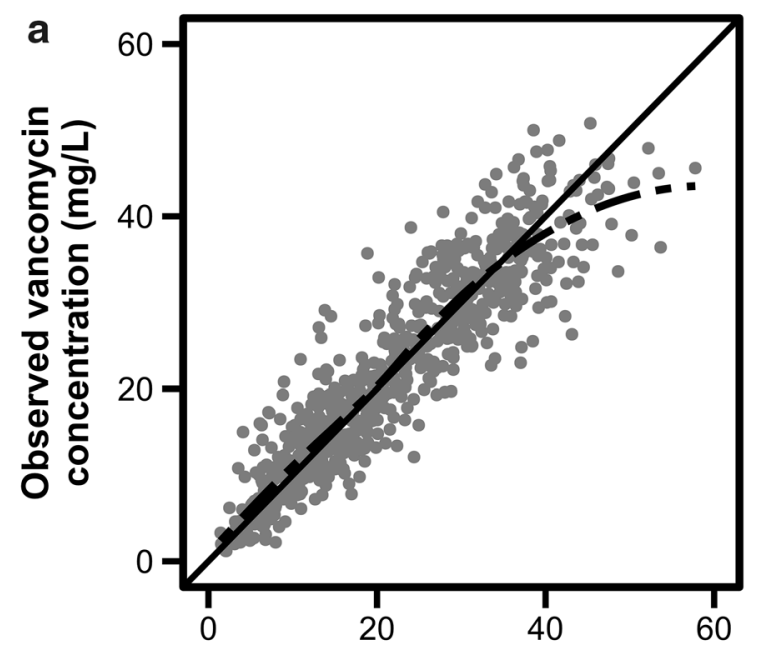

Population predicted vancomycin concentration (mg/L)

Fig. 1 An external evaluation of the predictive performance of a previously published neonatal vancomycin population pharmacokinetic model [7]. a Diagnostic plot depicting the model fit for observed versus population predicted vancomycin concentrations. The dashed black line reasonable with a median absolute prediction error of $3.1(95 \% \quad \mathrm{CI}: \quad 2.7-3.2) \mathrm{mg} / \mathrm{L}$. The predictive performance of the model for peak and trough concentrations is featured in Table 3. When incorporating patient concentrations to obtain Bayesian estimates of pharmacokinetic parameters for each neonate, the precision of the model predicted vancomycin concentrations (IPRED) improved (Table 4). For example, the median IPRED absolute prediction error was 1.7 (95\% CI: 1.5-1.8) $\mathrm{mg} / \mathrm{L}$.

Simulation-based diagnostics of the vancomycin pharmacokinetic model demonstrated a mean NPDE of 0.05 and a variance of 0.96 , indicating no bias and an ability of the model to reasonably capture the underlying variability in the external validation cohort. Additionally, there were no trends in NPDE across weight, postmenstrual age, serum creatinine, or time after dose (Fig. 2).

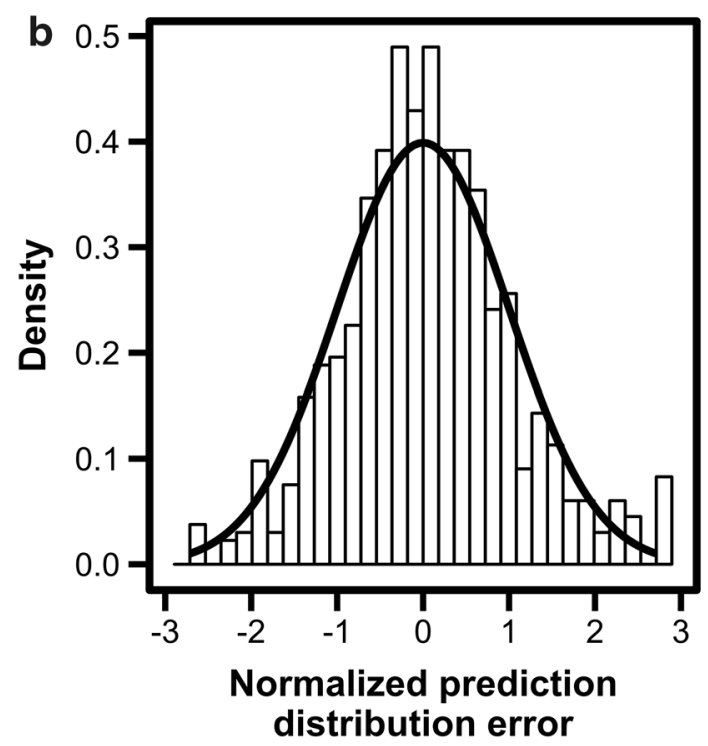

represents the locally weighted scatterplot smoothed fit of the data. $\mathbf{b}$ Kernel density plot of the normalized prediction distribution errors with a histogram depicting a normal, Gaussian distribution overlaid for comparative purposes 
Table 3 Predictive performance of the neonatal population pharmacokinetic model in the external validation cohort

\begin{tabular}{llll}
\hline Predictive measure & All concentrations & First peak & First trough \\
\hline Prediction error & & & -0.1 \\
Median & -0.8 & -2.0 & -0.5 to 0.2 \\
$95 \%$ confidence interval & -1.4 to -0.4 & -2.9 to -1.4 & $-1.5 \%$ \\
Percent prediction error & & $-7.5 \%$ & $-4.5 \%$ to $2.7 \%$ \\
Median & $-4.5 \%$ & $-9.4 \%$ to $-4.9 \%$ & \\
95\% confidence interval & $-7.2 \%$ to $-2.2 \%$ & & 2.1 \\
Absolute prediction error & & 3.9 & 1.7 to 2.7 \\
Median & 3.0 & 3.4 to 4.1 & \\
95\% confidence interval & 2.7 to 3.5 & & $20.1 \%$ \\
Absolute percent prediction error & & $12.6 \%$ & $16.8 \%$ to $24.0 \%$ \\
Median & $15.2 \%$ & $10.9 \%$ to $14.4 \%$ & \\
95\% confidence interval & $14.1 \%$ to $17.3 \%$ & &
\end{tabular}

Table 4 Predictive performance of the neonatal population pharmacokinetic model in the external validation cohort after incorporating patient drug concentrations in predictions (e.g., IPRED method)

\begin{tabular}{llll}
\hline Predictive measure & All concentrations & First peak & First trough \\
\hline Prediction error & & & -0.2 \\
Median & -0.7 & -1.7 & -0.4 to 0.1 \\
$95 \%$ confidence interval & -0.9 to -0.5 & -2.2 to -1.4 & $-1.7 \%$ \\
Percent prediction error & & $-5.8 \%$ & $-3.4 \%$ to $0.6 \%$ \\
Median & $-3.8 \%$ & -7.5 to $-4.6 \%$ & \\
$95 \%$ confidence interval & $-4.9 \%$ to $-3.2 \%$ & & 0.9 \\
Absolute prediction error & & 2.7 & $0.7-1.1$ \\
Median & 1.7 & $2.1-3.1$ & \\
$95 \%$ confidence interval & 1.5 to 1.8 & & $9.1 \%$ \\
Absolute percent prediction error & & $8.4 \%$ & $7.3 \%$ to $10.8 \%$ \\
Median & $8.8 \%$ & $7.3-9.6 \%$ & \\
$95 \%$ confidence interval & $8.1-9.7 \%$ & &
\end{tabular}

Trough Concentration and $\mathrm{AUC}_{24}$ Relationship

A linear relationship between increased $\mathrm{AUC}_{24}$ and higher trough concentrations was observed in the external validation cohort $\left(r^{2}=0.60\right.$; Fig. 3a). AUC 24 was highly variable at a given trough concentration (i.e., a two- to threefold range of $\mathrm{AUC}_{24}$ was achieved at a given trough concentration), and therefore, $\mathrm{AUC}_{24}$ could not 

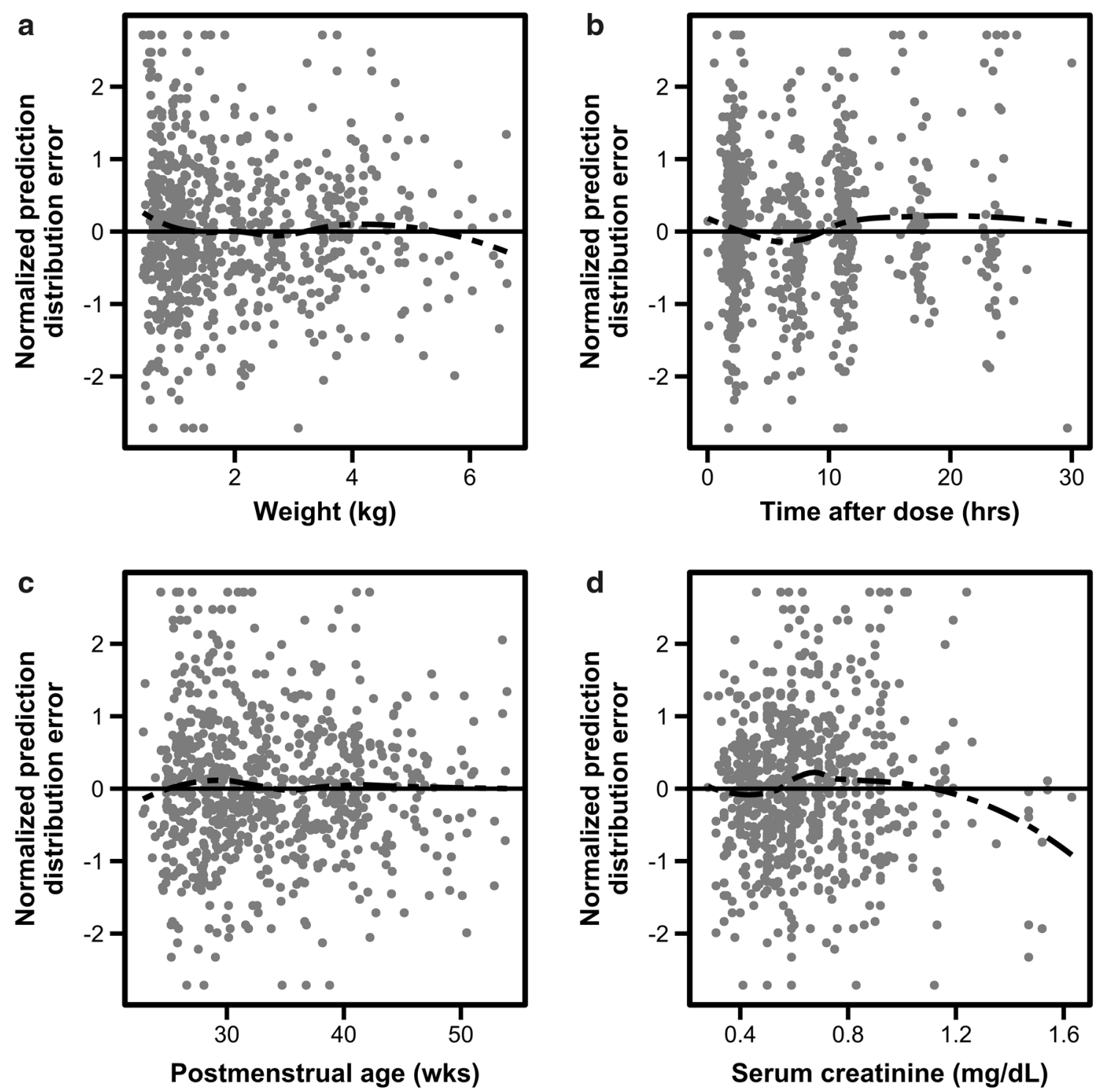

Fig. 2 Assessment of the predictive performance of the neonatal vancomycin population pharmacokinetic model. a Normalized prediction distribution errors versus weight, measured in kilograms. b Normalized prediction distribution errors versus the time elapsed since the last vancomycin dose, measured in hours. c Normalized prediction

be precisely predicted for an individual neonate based on a trough concentration alone. However, a trough concentration of $11 \mathrm{mg} / \mathrm{L}$ predicted the achievement of an $\mathrm{AUC}_{24} \geq 400$ in 93\% of neonates (Fig. 3b). The median (range) $\mathrm{AUC}_{24}$ at this trough concentration was 542 (308 to 649$) \mathrm{mg} \times \mathrm{hr} / \mathrm{L}$.

distribution errors versus postmenstrual age, measured in weeks. d Normalized prediction distribution errors versus serum creatinine concentrations, measured in milligrams per deciliter. The dashed black lines represent locally weighted scatterplot smoothed fits of the data

\section{DISCUSSION}

External validation of a population pharmacokinetic model is described by the United States Food and Drug Administration as "the most stringent method for testing a developed model" [16]. Yet, external validation 


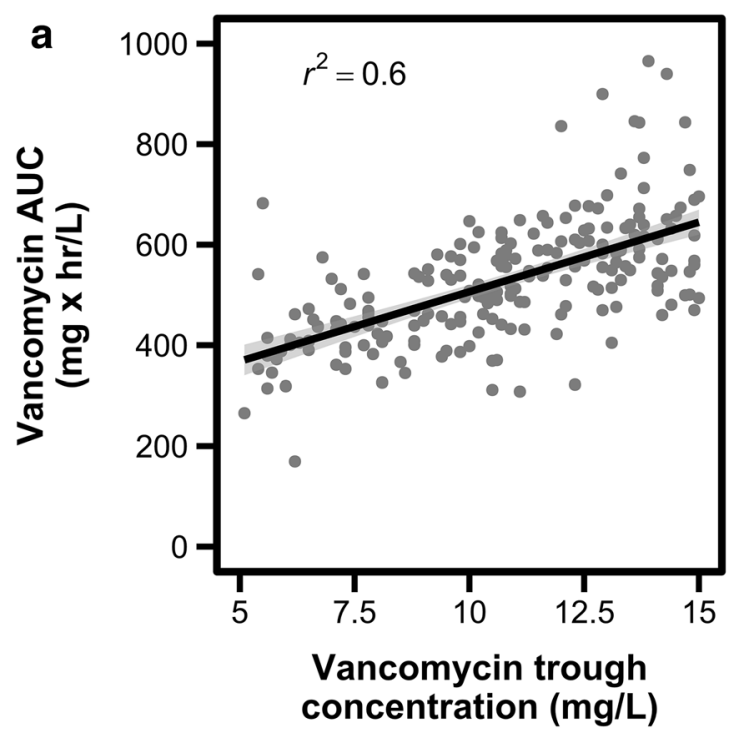

Fig. 3 The association between vancomycin trough concentrations and the extent of drug exposure, as measured by the 24-h area under the curve $\left(\mathrm{AUC}_{24}\right)$. a Higher vancomycin trough concentrations were associated with higher $\mathrm{AUC}_{24}$ values, although substantial variability was noted. b The probability of achieving a pharmacokinetic/ pharmacodynamic target associated with clinical and

is performed in $<10 \%$ of published pharmacokinetic models and concerns about the clinical utility of the model often remain [17]. The external validation performed in the current study strengthens a previously published neonatal vancomycin population pharmacokinetic model. Namely, we found the pharmacokinetic model to be unbiased across the largest cohort of neonates used in a validation study to date. The precision of the model when utilizing only a neonate's postmenstrual age, weight, and serum creatinine was $12.6 \%$ for peak concentrations and $20.1 \%$ for trough concentrations. When a neonate's drug concentrations are incorporated into the model (such as would occur after therapeutic drug monitoring in the NICU), the precision further improved to 8.4 and $9.1 \%$ for peak and trough concentrations, respectively. This level of precision suggests that the model

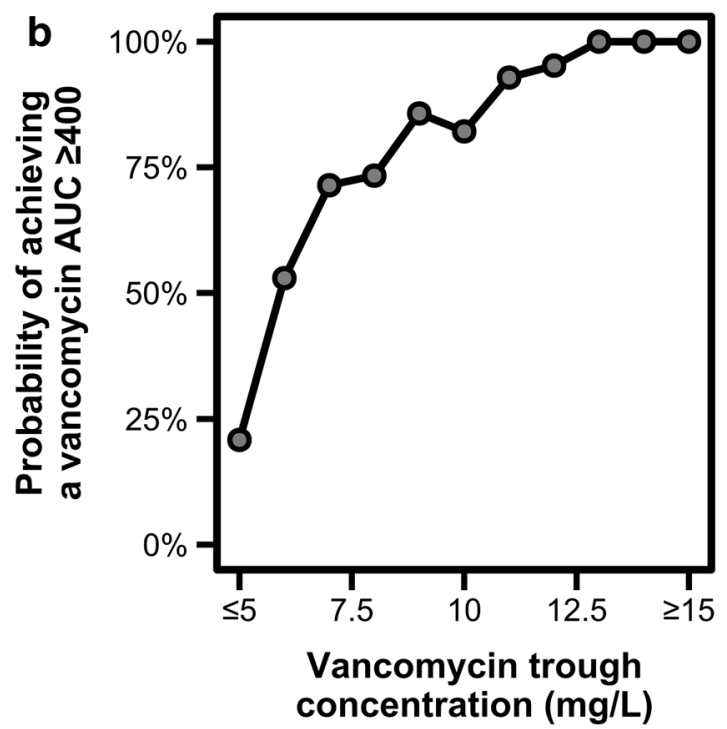

microbiological success for invasive methicillin-resistant Staphylococcus aureus infections (an $\mathrm{AUC}_{24} \geq 400$ ) increased with higher vancomycin trough concentrations. All neonates with a trough $\geq 12 \mathrm{mg} / \mathrm{L}$ had an $\mathrm{AUC}_{24} \geq 400$, although many neonates achieved the $\mathrm{AUC}_{24}$ target with lower trough concentrations

may be useful in evaluating vancomycin dosing regimens and estimating the extent of drug exposure in the clinical setting.

A recent clinical study by Ringenberg et al. [4] highlights the current challenges with vancomycin dosing in neonates. In a multicenter retrospective evaluation, vancomycin dosing guidelines from Neofax resulted in only $25 \%$ of the neonates studied achieving a target trough concentration of 10-20 mg/L with empiric dosing [4]. Moreover, the authors reported that $20 \%$ of the neonates included in their study had a trough concentration $<5 \mathrm{mg} / \mathrm{L}$ (Theresa Ringenberg, personal communication, April 15, 2015). Even after therapeutic drug monitoring and dose adjustment, only $45 \%$ of neonates achieved the goal trough concentration of $10-20 \mathrm{mg} / \mathrm{L}$ at any point during their course of therapy. This study clearly reveals the 
significant clinical challenge associated with reliably achieving therapeutic and safe vancomycin concentrations in this highly variable patient population [4]. More innovative vancomycin dosing strategies and approaches are needed in neonates that can help providers personalize empiric dose selection, interpret therapeutic drug monitoring data, and adjust dosing so that exposure targets are achieved.

Population pharmacokinetic models are a powerful tool that can aid clinicians and help inform dosing decisions [18, 19]. By incorporating patient-specific characteristics, dosing information, drug concentrations, and consideration of the variability between patients, population pharmacokinetic models offer the opportunity to provide a more personalized approach to therapeutic decision making. This is especially valuable in a highly variable population, such as neonates, receiving a narrow therapeutic window drug such as vancomycin.

In adults, Bayesian approaches utilizing population pharmacokinetic models have already been shown to have the potential to help support vancomycin dosing decisions [19, 20]. Advancement of similar approaches in neonates is needed. The development and external validation of a neonatal vancomycin population pharmacokinetic model lays the foundation for this future work. For example, our group is currently developing a modelbased approach to individualize the empiric dose in neonates that incorporates the predictors of weight, postmenstrual age, and serum creatinine. Using a simulation framework, the vancomycin dose for a given neonate that is most likely to achieve an $\mathrm{AUC}_{24}$ $\geq 400$ while still maintaining a trough concentration $<20 \mathrm{mg} / \mathrm{L}$ is calculated. A userfriendly, web-based application is currently being developed to facilitate the adoption of this model in our neonatal intensive care units, including integration into the electronic health record. In addition, the ability to estimate $\mathrm{AUC}_{24}$ and assist providers with dose adjustment within the clinical workflow would be of high value.

Until more robust clinical dosing support tools are developed, clinicians will continue to rely on trough concentration monitoring to help guide vancomycin dosing in neonates. Our findings reinforce the large variability observed in vancomycin trough concentrations among neonates and the inability of a trough concentration alone to reliably predict an individual neonate's $\mathrm{AUC}_{24}$. Targeting an $\mathrm{AUC}_{24} / \mathrm{MIC} \geq 400$ is recommended by the Infectious Disease Society of America when treating invasive MRSA infections and a trough concentration of $15-20 \mathrm{mg} / \mathrm{L}$ is suggested in adults to achieve this target [1, 21]. The current study provides further support that in neonates a vancomycin trough concentration of $15-20 \mathrm{mg} / \mathrm{L}$ is unnecessary to achieve an $\mathrm{AUC}_{24} / \mathrm{MIC} \geq 400$ with an $\mathrm{MIC}$ $\leq 1 \mathrm{mg} / \mathrm{L}$ and that lower trough concentrations are likely adequate based on $\mathrm{AUC}_{24}$ considerations [7]. Accordingly, a trough concentration of approximately $10 \mathrm{mg} / \mathrm{L}$ is likely a reasonable first-line target that will provide adequate exposure for invasive MRSA while also appropriately covering for coagulase negative staphylococcal infections. Further dose adjustment and individualization of the therapeutic approach should be guided by the specific pathogen identified, susceptibility testing, clinical status, etc. For example, for MRSA infections with MICs $\geq 2 \mathrm{mg} / \mathrm{L}$, an alternative to vancomycin may be necessary since an $\mathrm{AUC}_{24} / \mathrm{MIC} \geq 400$ will not be achieved in neonates even at trough concentrations of 15-20 mg/L [7]. Lastly, the extent to which the 
target $\mathrm{AUC}_{24} / \mathrm{MIC} \geq 400$ is generalizable to neonates is unclear and requires further study.

\section{CONCLUSIONS}

In summary, an evaluation of a recently published neonatal vancomycin population pharmacokinetic model in a large external dataset supported the predictive performance and generalizability of the model. The model may be useful in evaluating vancomycin dosing regimens and for estimating the extent of drug exposure in neonates.

\section{ACKNOWLEDGMENTS}

The authors received financial support from the American Foundation for Pharmaceutical Education, Primary Children's Hospital Foundation, and the National Institutes of Health, which supported their effort spent conducting this study as noted in the conflict of interest section. No funding or sponsorship was received for the publication of this article. All named authors meet the International Committee of Medical Journal Editors (ICJME) criteria for authorship for this manuscript, take responsibility for the integrity of the work as a whole, and have given final approval for the version to be published.

Conflict of interest. CS is supported by the American Foundation for Pharmaceutical Education's Clinical Pharmaceutical Sciences Fellowship. JKR is supported by the Pharmacotherapy Subspecialty Award from the Primary Children's Hospital Foundation. AF is supported by the National Institutes of Health (NICHD 1K23HD079557). ALH is supported by the National Institutes of Health (NICHD
K08HS023320). JB, EKK, MGS, and CMTS declare that they have no conflicts of interest.

Compliance with ethics guidelines. All procedures followed were in accordance with the ethical standards of the responsible committee on human experimentation (institutional and national) and with the Helsinki Declaration of 1975, as revised in 2013. A waiver of informed consent was granted by the Institutional Review Boards of the University of Utah and Primary Children's Hospital for the retrospective data presented in this study.

Open Access. This article is distributed under the terms of the Creative Commons Attribution Noncommercial License which permits any noncommercial use, distribution, and reproduction in any medium, provided the original author(s) and the source are credited.

\section{REFERENCES}

1. Liu C, Bayer A, Cosgrove SE, et al. Clinical practice guidelines by the infectious diseases society of america for the treatment of methicillin-resistant Staphylococcus aureus infections in adults and children. Clin Infect Dis An Off Pub Infect Dis Soc Am. 2011;52(3):e18-55.

2. Kearns GL, Abdel-Rahman SM, Alander SW, Blowey DL, Leeder JS, Kauffman RE. Developmental pharmacology-drug disposition, action, and therapy in infants and children. New Eng J Med. 2003;349(12):1157-67.

3. Stockmann C, Roberts JK, Yu T, et al. Vancomycin pharmacokinetic models: informing the clinical management of drug-resistant bacterial infections. Expert Rev Anti Infect Ther. 2014;12(11):1371-88.

4. Ringenberg T, Robinson C, Meyers R, et al. Achievement of therapeutic vancomycin trough serum concentrations with empiric dosing in neonatal intensive care unit patients. Pediat Infect Dis J; 2015; (Epub ahead of print). 
5. Vandendriessche A, Allegaert K, Cossey V, Naulaers G, Saegeman V, Smits A. Prospective validation of neonatal vancomycin dosing regimens is urgently needed. Curr Ther Res Clin Exp. 2014;76:51-7.

6. Jacqz-Aigrain E, Zhao W, Sharland M, van den Anker JN. Use of antibacterial agents in the neonate: 50 years of experience with vancomycin administration. Sem Fetal Neo Med. 2013;18(1):28-34.

7. Frymoyer A, Hersh AL, El-Komy $\mathrm{MH}$, et al. Association Between Vancomycin Trough Concentration and AUC in Neonates. Antimicr Agents Chemother; 2014.

8. Sheiner LB, Beal S, Rosenberg B, Marathe VV. Forecasting individual pharmacokinetics. Clin Pharmacol Ther. 1979;26(3):294-305.

9. Sheiner LB, Rosenberg B, Melmon KL. Modelling of individual pharmacokinetics for computer-aided drug dosage. Comput Biomed Res Int J. 1972;5(5):411-59.

10. Sheiner LB, Beal SL. Bayesian individualization of pharmacokinetics: simple implementation and comparison with non-Bayesian methods. J Pharm Sci. 1982;71(12):1344-8.

11. Schumacher GE, Barr JT. Bayesian approaches in pharmacokinetic decision making. Clin Pharm. 1984;3(5):525-30.

12. Srivastava T, Alon US, Althahabi R, Garg U. Impact of standardization of creatinine methodology on the assessment of glomerular filtration rate in children. Pediatr Res. 2009;65(1):113-6.

13. Sheiner LB, Beal SL. Some suggestions for measuring predictive performance. J Pharmacokinet Biopharm. 1981;9(4):503-12.

14. Brendel K, Comets E, Laffont C, Laveille C, Mentre F. Metrics for external model evaluation with an application to the population pharmacokinetics of gliclazide. Pharm Res. 2006;23(9):2036-49.
15. Brendel K, Comets E, Laffont C, Mentre F. Evaluation of different tests based on observations for external model evaluation of population analyses. J Pharmacokinet Pharmacodyn. 2010;37(1):49-65.

16. US FDA. Guidance for industry: population pharmacokinetics [online]. Available from: http:// www.fda.gov/downloads/Drugs/Guidances/UCM07 2137.pdf. Accessed 18 Sep 2014.

17. Brendel K, Dartois C, Comets E, et al. Are population pharmacokinetic and/or pharmacodynamic models adequately evaluated? A survey of the literature from 2002 to 2004. Clin Pharmacokinet. 2007;46(3):221-34.

18. Lee JY, Garnett CE, Gobburu JV, et al. Impact of pharmacometric analyses on new drug approval and labelling decisions: a review of 198 submissions between 2000 and 2008. Clin Pharmacokinet. 2011;50(10):627-35.

19. Neely MN, Youn G, Jones B, et al. Are vancomycin trough concentrations adequate for optimal dosing? Antimicrob Agents Chemother. 2014;58(1):309-16.

20. Nunn MO, Corallo CE, Aubron C, Poole S, Dooley MJ, Cheng AC. Vancomycin dosing: assessment of time to therapeutic concentration and predictive accuracy of pharmacokinetic modeling software. Annal Pharmacother. 2011;45(6):757-63.

21. Rybak MJ, Lomaestro BM, Rotschafer JC, et al. Vancomycin therapeutic guidelines: a summary of consensus recommendations from the infectious diseases Society of America, the American Society of Health-System Pharmacists, and the Society of Infectious Diseases Pharmacists. Clin Infect Dis An Off Pub Infect Dis Soc Am. 2009;49(3):325-7. 